\title{
Neurochemical Effects of Vitamins C, E and DMSO Combinations on Oxidative Stress Biomarkers and Severity of Ischemic Stroke in Wistar Rats
}

\author{
Suleiman ${ }^{1 *}$, Bulama $\mathrm{I}^{2}$, Muhammad $\mathrm{NI}^{1}$, Aishat $\mathrm{DI}^{3}$, Balarabe $\mathrm{SA}^{4}$, Ngaski AA ${ }^{5}$, Buhari $\mathrm{S}^{6}$, Jimoh AA ${ }^{7}$, Abbas \\ $\mathrm{AY}^{3}$, Saidu $\mathrm{Y}^{3}$ and Bilbis $\mathrm{LS}^{3}$
}

${ }^{1}$ Department of Veterinary Physiology and Biochemistry, Nigeria

${ }^{2}$ Department of Veterinary Physiology and Biochemistry, University of Maiduguri, Nigeria

${ }^{3}$ Department of Biochemistry, Nigeria

${ }^{4}$ Department of Medicine, College of Health Science, Nigeria

${ }^{5}$ Department of Chemical Pathology, Nigeria

${ }^{6}$ Department Veterinary Surgery and Radiology, Nigeria

${ }^{7}$ Department of Theriogenology and Animal Production, Usmanu Danfodiyo University, Sokoto, Nigeria

*Corresponding author: Suleiman Nasiru, Department of Veterinary Physiology

and Biochemistry, Nigeria.

Received Date: September 06, 2018

Published Date: October 01, 2018

\begin{abstract}
Objective: The objective of this research is to reports the effect of DMSO, Vitamins C and combinations on the oxidative stress biomarkers severity of induced ischemic stroke (IS) in Wistar rats.
\end{abstract}

Materials and methods: Twenty-five apparently healthy rats were divided into five groups of five rats each. Ischemic stroke was induced using middle cerebral artery occlusion (MCAO) method, $45 \mathrm{mg} / \mathrm{kg}$ body weight of the combinations (Vitamin C, Vitamin E and DMSO) were orally administered to the rats for two weeks, antioxidant enzymes (catalase (CAT) superoxide dismutase (SOD) and glutathione peroxide (GPX) activities and oxidative stress biomarkers (thiobarbituric acid reactive species (TBARS) concentration, were assessed.

Results: Ischemic stroke caused significantly $(\mathrm{p}<0.05)$ decrease in the activities of the enzymes and significant increase $(\mathrm{P}>0.05)$ in the concentration of TBARS. Treatment with $45 \mathrm{mg} / \mathrm{kg}$ of the antioxidant combinations resulted in the significant increase $(\mathrm{P}<0.05)$ of the activities of CAT, SOD and GPX. Also, there was significant $(\mathrm{p}<0.05)$ decrease in the concentration of TBARS.

Conclusion: The combinations of DMSO, Vitamins C and E reduced oxidative stress and its biomarkers in induced ischemic stroke in rats and underscores the relevance of antioxidants in the treatment of IS.

Key words: Ischemic stroke; Antioxidants; Oxidative stress

Abbreviation: DMSO: Dimethyl Sulfoxide; IS: Ischemic Stroke; MCAO: Middle Cerebral Artery Occlusion; SOD: Superoxide Dismutase; CAT: Catalase; GPX: Glutathione Peroxidase; TBARS: Thiobarbiturate acid Reactive Species; ROS: Reactive Oxygen Species; OS: Oxidative Stress; CT: Computed Tomography; MRI: Magnetic Resonance Imaging

\section{Introduction}

Stroke is a growing disease and is the third commonest cause of death in the world after coronary heart disease and cancer, especially in the elderly [1]. In Nigeria, Stroke constitutes about $2.4 \%$ of all emergency admissions with cerebral infarction making up to $49 \%$ of all cases [2]. The burden of Stroke in Nigeria is such that it is responsible for $1.8 \%$ of all deaths in the emergency unit with case fatality rates that increase from $9 \%$ to $46 \%$, depending on the duration of the event [2].

Stroke treatment is dependent on whether it is ischaemic or haemorrhagic, and therefore diagnosis within the first six hours after onset of symptoms is essential if patients with ischaemic stroke are to benefit from t-PA therapy since it is the only FDA 
approved treatment for stroke. Standard neuroimaging techniques, such as computed tomography (CT) and magnetic resonance imaging (MRI), and models based on clinical data have all been evaluated in the diagnosis of stroke.

It is evident that reactive oxygen species (ROS) as well as the downstream activated cytokines may play major roles in IR injury [3]. ROS are oxygen-containing reactive molecules that can be naturally generated from biological systems. When produced at low levels, ROS act as important signaling molecules involved in a variety of physiological activities such as immune response, muscle contraction, and exercise adaptation [4].

Endogenous antioxidants including catalase, superoxide dismutase (SOD), glutathione, and glutathione peroxidase (GPX) are important ROS scavengers that are responsible for maintaining ROS at normal levels. However, under specific pathological conditions, antioxidant defenses can be overwhelmed, resulting in cellular oxidative stress [4].

Cerebral ischemia and reperfusion (I/R) injury is a complex of pathophysiological events linked to deprivaition of oxygen and energy from blood during the period of ischemia, eventually resulting in neuronal destruction [5]. Pathogenic mechanisms following cerebral ischemia and reperfusion are associated with energy failure, elevation of the intracellular $\mathrm{Ca} 2+$ level, release of excitatory neurotransmitters, oxidative stress, inflammation, apoptosis, etc. [6].

Currently, there are two therapeutic strategies used in cerebral ischemia and reperfusion (I/R) injury. The most common is to restore blood flow by administering thrombolytic agents [7]. However, thrombolytic therapy can lead to hemorrhagic complications, and the strict 3-hour time therapeutic window is the main barrier. Neuroprotection, as another strategy for treatment of cerebral ischemia and reperfusion injury, has attracted much attention in recent years [8]. Accumulating evidence has shown that antioxidants exert beneficial effects in a variety of neurodegenerative disorders, including traumatic brain injury, ischemic stroke etc [9].

\section{Materials and Methods}

\section{Animals and treatment}

Twenty-five apparently healthy rats of Wistar strain weighing between 180 and 200g were obtained from the Animal House of the Department of Veterinary Physiology and Biochemistry, Faculty of Veterinary Medicine, Usmanu Danfodiyo University, Sokoto, Nigeria. The Ethics Committee of the Department of Veterinary Physiology and Biochemistry approved the animal experiment with ethical clearance number VPB/EC/17/16. The rats were housed under a standard condition at room temperature of $35-37.5{ }^{\circ} \mathrm{C}$ and were subjected to a 12-hour light/12-hour dark cycle. The rats were fed with a grower mash of vital feed ad-libitum. They were randomly divided into five groups of five rats each as shown in Table 1 below. The treatment begins 12 hours post IS induction after the rats have recovered from anesthesia.

\section{Stroke induction}

The focal cerebral ischemic model was conducted using MCAO method as described by Spratt et al. [10]. IS was induced in this study by occluding the middle cerebral artery (MCA) in win star rats. Ketamine and Xylazine at the dose of $80 \mathrm{mg} / \mathrm{kg}$ and $5 \mathrm{mg} / \mathrm{kg}$ body weight respectively were used to anaesthize the rats. The anesthetic condition was maintained until the end of the occluding period. The neck regions of the rats were shaved and scrubbed with savlon, incision was done at the scrubbed area to gain access to the common carotid artery (CCA). The artery was ligated proximally, a nitch incision was created on the CCA distally using a 25G needle, and absorbable suture material chromic catgut was inserted through the nitch incision into the artery until resistance was felt. A silicon-coated suture with the diameter and length of $0.35 \mathrm{~mm}$ and $5 \mathrm{~mm}$, respectively was manipulated through the external and internal carotid arteries to block the MCA. The incisions made were closed using a non-absorbable suture material (nylon) and the rats were allowed to recover from the anaesthetic condition in the cages. During the induction, the heart rate and rectal temperatures were monitored (Table 1 ).

Table 1: Experimental design.

\begin{tabular}{|c|c|}
\hline Group & Antioxidants Treatment \\
\hline I & Nonstroke non-treated (NSNT) \\
\hline II & Stroke induced non-treated (SNT) \\
\hline III & $\begin{array}{c}\text { Stroke induced and treated with } \\
\text { combinations of vitamins C and E } \\
\text { (Vit C+E) }\end{array}$ \\
\hline IV & $\begin{array}{c}\text { Stroke induced and treated with } \\
\text { combinations of vitamins C and } \\
\text { DMSO (Vit C+DMSO) }\end{array}$ \\
\hline V & $\begin{array}{c}\text { Stroke induced and treated with } \\
\text { combinations of vitamins C, E and } \\
\text { DMSO (Vit C+E+DMSO) }\end{array}$ \\
\hline
\end{tabular}

\section{Blood sample collection}

Following the two weeks of the antioxidant treatment to the experimental rats, blood samples were collected from the rats through cardiac puncture. The rats were anaesthetized using chloroform in a glass jar. After proper anesthesia the rats were laid on dorsal recumbency and then the needle was inserted between the intercostal muscles to gain access to the heart. Blood was collected and poured into plain tubes and spun with centrifuge at 3000rpm for 5 minutes using a bench top centrifuge, the supernatant was removed and stored at $-20{ }^{\circ} \mathrm{C}$ until required for analyses for oxidative stress indices.

\section{Biochemical analyses}

Estimation of Catalase (CAT) activity: The commercial Cayman's Catalase Assay Kit was obtained from the Cayman chemical company, Ann Arbor, USA and was used to estimate activities of serum catalase in according to the manufacturer's instructions according to the method of Johnsson \& Borg [11]. Three wells were designated as a sample, standardization and control. To each well, $100 \mu \mathrm{l}$ of assay buffer and $30 \mu$ l of methanol 
were added. To standard well, $20 \mu \mathrm{l}$ of prepared standard (Formaldehyde Standards) were added and to sample well $20 \mu \mathrm{l}$ of serum were added. $20 \mu \mathrm{l}$ of $\mathrm{H} 202$ were added to each well to initiate the reaction. The plate was covered with a lid and incubated on a shaker for 20 minutes at room temperature. To each well, $30 \mu \mathrm{l}$ of potassium hydroxide were added to terminate the reaction and $30 \mu \mathrm{l}$ of purpald were then added. The plate was covered once again and incubated for 10 minutes at room temperature on a shaker. Once again, to each well, $10 \mu$ l of potassium periodate were added, covered and incubated for 5 minutes on a shaker. The absorbance was read at $540 \mathrm{~nm}$ using Rayto RT $2100 \mathrm{C}$ plate reader produced by Shanghai International Holding Corporate, Humberg, Germany.

Estimation of Superoxide Dismutase (SOD) activity: Cayman's Superoxide Dismutase Assay Kit from Cayman chemical company, Ann Arbor, USA was used according to the manufacturer's instructions to quantify the serum activities of superoxide dismutase. Two wells were designated as standard and sample. To each well $200 \mu \mathrm{l}$ of the diluted radical detector, $10 \mu \mathrm{l}$ each of the prepared standard to the serum were added to the standard well and sample well respectively. Twenty microliter $(20 \mu \mathrm{l})$ of diluted xanthine oxidase was added to both standard and sample wells to initiate the reaction. The plate was shaken for a few seconds and covered with cover plate. The plate was then incubated on a shaker at room temperature for 20 minutes and absorbance was read at 450nm using Rayto RT 2100C plate reader produced by Shanghai International Holding Corporate, Humberg, Germany.

With the method described by Marklund [12].

Estimation of Glutathione Peroxidase (GPx) activity: The commercial assay kits of Cayman obtained from the Cayman chemical company, Ann Arbor, USA was used to carry out the analysis of GPx in accordance with the manufacture's guide. In brief, three wells were designated as sample, non-enzymatic and positive control. To sample well, $100 \mu \mathrm{l}$ of assay buffer, $50 \mu \mathrm{l}$ of cosubstrate mixture and $20 \mu \mathrm{l}$ of serum were added. To non-enzymatic well, $120 \mu \mathrm{l}$ of assay buffer and $50 \mu \mathrm{l}$ of co-substrate mixture were added and to positive control well $100 \mu$ l of assay buffer, $50 \mu \mathrm{l}$ of co-substrate mixture and $20 \mu \mathrm{l}$ of diluted GPx were added. The reaction was initiated by adding $20 \mu \mathrm{l}$ of cumene hydroperoxide to each well and the plate was carefully shaken for a few seconds. The absorbance was read at $340 \mathrm{~nm}$ using Rayto RT 2100C plate reader produced by Shanghai International Holding Corporate, Humberg, Germany, once every 3 minutes according to the method described by Paglia \& Valentine [13].

$$
\begin{aligned}
\triangle \mathrm{Abs} / \mathrm{min} & =\frac{\text { Abs }(\text { time } 2)-\text { Abs }(\text { time } 1)}{\text { Time } 2(\mathrm{~min})-\text { Time } 1(\mathrm{~min})} \\
\text { GPx activity } & =\frac{A b s / \mathrm{min}}{0.00373 \mu / \mathrm{M}} \times \frac{0.19 \mathrm{ml}}{0.02 \mathrm{ml}}=\mathrm{nmol} / \mathrm{mn} / \mathrm{ml}
\end{aligned}
$$

Estimation of Lipid Peroxidation (MDA) concentration: Estimation of lipid peroxidation was performed using Cayman's assay kits obtained from the Cayman chemical company, Ann Arbor, USA, following the manufacture's instruction. Into a test tube, $0.1 \mathrm{ml}$ of serum was added and treated with $2 \mathrm{ml}$ of TBA-TCA-HCl (1:1:1 ratio) reagent (Thiobarbituric acid $0.37 \%, 0.25 \mathrm{~N} \mathrm{HCl}$ and $15 \%$ TCA). The tube was placed in water-bath for 15 minutes, cooled and centrifuged at room temperature for 10 minutes at 1000 RPM. The absorbance of clear supernatant was measured against reference blank at $535 \mathrm{~nm}$ using Rayto RT 2100C plate reader produced by Shanghai International Holding Corporate, Humberg, Germany. The concentration of TBARS was calculated using the molar extinction coefficient of malondialdehyde ( $1.5 \times 105 \mathrm{Mol} / \mathrm{l} / \mathrm{cm})$ following the method highlighted by Niehans \& Samuelson [14].

Data analysis: Statistical programmed in Social Sciences (SPSS 22.0) was used to analyse the data. Results were expressed as means \pm SD. The data were analyzed by one-way analysis of variance (ANOVA); Turkey Alpha Post-hoc Test was used for multiple comparisons between groups and concentrations.

\section{Results}

Figure 1 The effect of supplementation of antioxidants on the activity of catalase is presented in Figure 1. The results indicated that stroke caused significant $(\mathrm{p}<0.05)$ decrease in the activity of the enzyme as shown by stroke nontreated group (SNT). After been given the combinations of the various antioxidants at $45 \mathrm{mg} / \mathrm{kg} \mathrm{BW}$, the activity of CAT increased significantly $(\mathrm{P}<0.05)$ compared to the activity of CAT in NSNT and SNT rats.

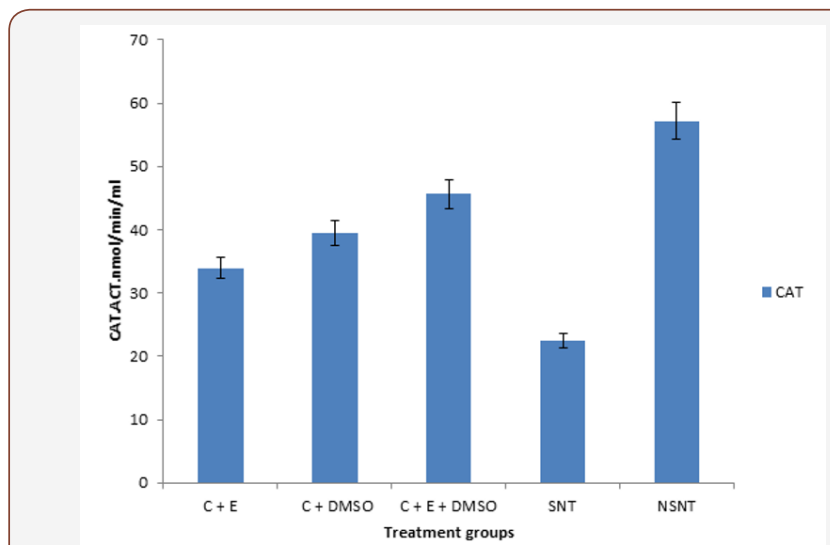

Figure 1: Effects of Antioxidants treatment on CAT activity of experimental rats.

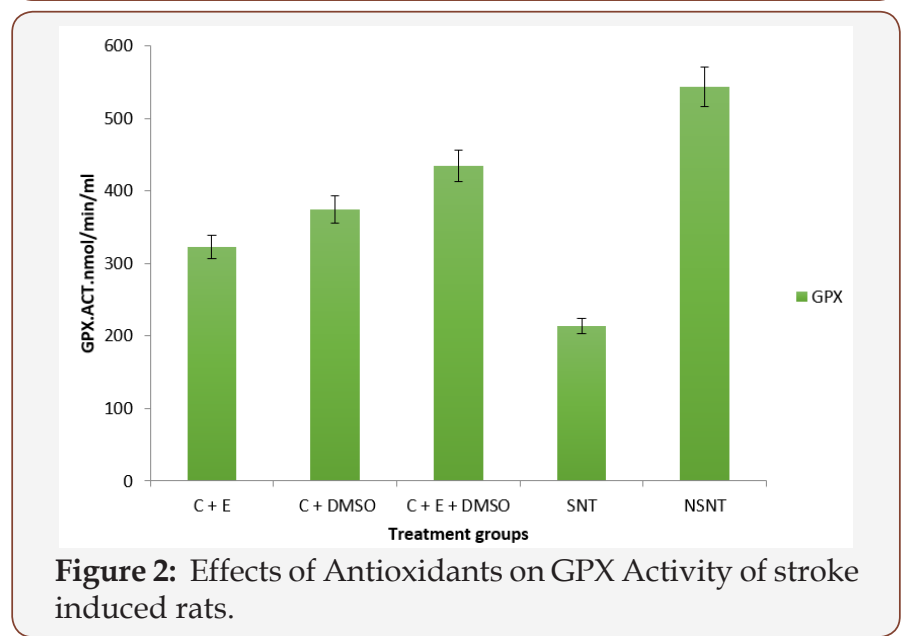

Figure 2 The effect of treatment of antioxidants on the activity of GPX is presented in Figure 2. The results indicated that stroke caused significant $(\mathrm{p}<0.05)$ decrease in the activity of the enzyme GPX as indicated by stroke nontreated group. After been treated with various combinations of antioxidants at $45 \mathrm{mg} / \mathrm{kg}$ BW, the activity of GPX changed significantly $(\mathrm{P}<0.05)$ when compared to SNT and NSNT groups. 


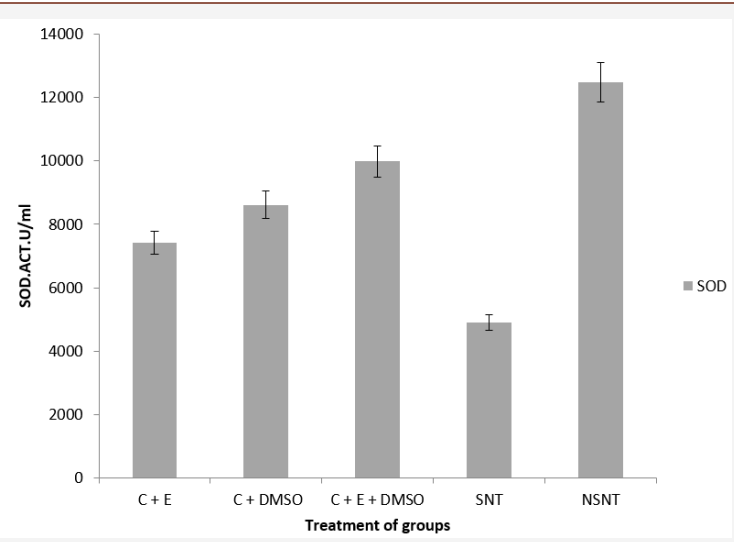

Figure 3: Effects of Antioxidants on SOD Activity of stroke induced rats.

Figure 3 The effect of treatment of antioxidants on the activity of SOD is presented in Figure 3. The results indicated that stroke caused significant $(\mathrm{p}<0.05)$ decrease in the activity of the enzyme SOD. After been treated with $45 \mathrm{mg} / \mathrm{kg}$ BW combinations of various antioxidants, the activity of SOD increased compared to the activity of SOD in SNT and NSNT rats.

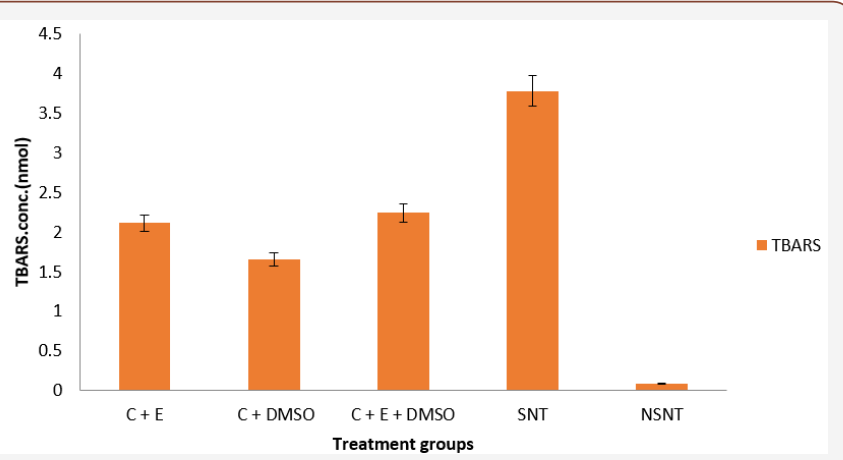

Figure 4: Effects of antioxidants on TBARS concentration of stroke induced rats.

Figure 4 The effects of the antioxidant treatment on the concentrations of Thiobarbituric Acid Reactive Substances (TBARS) are presented in Figure 4. The results indicated that stroke caused significant $(\mathrm{P}<0.05)$ increase in the concentrations of the TBARS in the SNT group. After the treatment with different combinations of antioxidants at $45 \mathrm{mg} / \mathrm{kg}$ body weight, the concentration of TBARS decreased significantly $(\mathrm{P}<0.05)$ compared to the concentration of TBARS in NSNT treated group of rats.

\section{Discussion}

Stroke is said to be a rapidly developing global neurological deficit lasting more than 24 hours or leading to death with no apparent cause other than vascular origin [15]. Oxidative stress (OS) has been implicated as a possible contributor to the pathogenesis of acute central nervous system (CNS) injury and it is found to be responsible for mortality following stroke [16]. In this study, three antioxidants (Vitamin C, Vitamin E and DMSO) were combined differently in the treatment of three groups (Vitamin $\mathrm{C}+$ Vitamin E, Vitamin C + DMSO, and Vitamin C + Vitamin E + DMSO). The observed significant $(\mathrm{P}<0.05)$ decrease in the activities of serum antioxidant enzymes; SOD, CAT, GPX and increased plasma TBARS concentration in all the stroke-induced-treated groups compared to the control groups (NSNT and SNT), suggested a role of oxidative stress in stroke. The imbalance between the endogenous antioxidants and reactive oxygen species produced may cause tissue damage, cell death by lipid peroxidation, carbohydrate oxidation, protein oxidation and nucleic acid oxidation, this is in agreement with the findings of [17]. Treatment of the stroke-induced rats with varying combinations of antioxidants (Vitamin C, Vitamin E and DMSO) caused a significant increase in stroke antioxidant status (SOD, CAT and GPX) with a decrease in TBARS concentration.

Vitamin C is found to be more abundant in tissues, where ROS production is more important, in addition vitamin $\mathrm{C}$ can regenerate vitamin $\mathrm{E}$ and GSH, which are very potent against ROS. The findings of this work are in agreement with the results of Rabec \& Pierce [18] (1994), who reported that ascorbate is highly efficient in trapping free radicals, and preventing them from forming lipid hydroperoxide that can be generated during the stroke. Eghwrudjakpor \& Allison [19] also reported that ascorbic acid is a very efficient free-radical scavenger because it neutralizes or removes the impurities formed by the free radicals, produced as a result of stroke. Inci et al. [20] reported that vitamin $\mathrm{E}$ is the most relevant chain-breaking antioxidant and abundance in cells and mitochondria membrane. It is also known that vitamin $\mathrm{E}$ acts directly on ROS, and it can also react with various antioxidants such as vitamin $\mathrm{C}, \mathrm{GSH}, \beta$-carotene to bring about synergistic activity, all these antioxidants mentioned have the ability to regenerate vitamin E, hence, Inci et al. [20] reported that vitamin $\mathrm{E}$ is promising in modifying OS pathways and improving neurological outcome in many animal studies. Vitamin E may also cause a neuroprotective effect by decreasing the rate of lipid peroxidation. According to Ramirez-Tortosa et al. [21], they reported that vitamin $\mathrm{E}$ is a lipid-soluble antioxidant which prevents the formation of lipid peroxide.

It was observed in this study that DMSO also played a significantly increased the activities of SOD, CAT and GPX enzymes, but it decreased the concentration of TBARS in the treated groups, when compared with the control groups. DMSO is widely used as a solvent for various drugs and it is reported to be an effective neuroprotectant. According to $\mathrm{Lu} \&$ Mattson [22], it averts glutamate-induced neuronal cell death. DMSO is reported to offer an option as a preventive measure in patients undergoing procedures with an increased risk of developing pre-Intserventional brain ischaemia, such as carotid coronary artery bypass surgery [23].

\section{Conclusion}

The combinations of DMSO, Vitamins $\mathrm{C}$ and $\mathrm{E}$ reduced oxidative stress and its biomarkers in induced ischemic stroke in rats and underscores the relevance of antioxidants in the treatment of IS.

\section{Acknowledgement}

None.

\section{Conflict of Interest}

No Conflict of Interest.

\section{References}

1. Llibre J, Valhuerdi A, Fernandez O, Llibre JC, Porto R, et al. (2010) Prevalence of stroke and associated risk factors in older adults in Havana 
city and Matanzas provinus, Cuba (10/66 population-based study). MEDICC Rev 12(3): 20-26.

2. Ogun SA, Ojini FI, Ogungbo B, Kolapo KO, Danesi MA (2005) Stroke in south west Nigeria. A 10-year review. Stroke 36(6): 1120-1122.

3. Gillani S, Cao J, Suzuki T, Hak DJ (2012) The effect of ischemia reperfusion injury on skeletal muscle. Injury 43(6): 670-675.

4. Zuo L, Zhou T, Pannell BK, Ziegler AC, Best TM (2015) Biological and physiological role of reactive oxygen species the good, the bad and the ugly. Acta Physiol (Oxf) 214(3): 329-348.

5. Khoshnam SE, Winlow W, Farzaneh M, Farbood Y, Moghaddam HF (2017) Pathogenic mechanisms following ischemic stroke. Neurol Sci 38(7): 1167-1186.

6. Durukan A, Tatlisumak T (2008) Acute ischemic stroke: overview of major experimental rodent models, pathophysiology, and therapy of focal cerebral ischemia. Pharmacol Biochem Behav 87(1): 179-197.

7. Saver JL (2011) Improving reperfusion therapy for acute ischaemic stroke. J Thromb Haemost 9: 333-343.

8. Patel RAG, McMullen PW (2017) Neuroprotection in the Treatment of Acute Ischemic Stroke. Prog Cardiovasc Dis 59(6): 542-548.

9. Suleiman N, Bilbis LS, Saidu Y, Nasiru JI, Dallatu MK, et al. (2015) Effect of some low molecular mass antioxidants in the management of traumatic brain injury in albino rats. Int J Curre Res 7(7): 18492-18499.

10.Spratt NJ, Fernandez J, Chen M, Rewell S, Cox S, et al. (2006) Modification of the method of thread manufacture improves stroke induction rate and reduces mortality after thread-occlusion of the middle cerebral artery in young or aged rats. Journal of Neuroscience Methods 155(2): 285-290.

11.Johansson LH, Borg LA (1988) A spectrophotometric method for determination of catalase activity in small tissue samples. Anal Biochem 174 (1): 331-336.

12. Marklund S (1980) Distribution of CuZn superoxide dismutase and Mn superoxide dismutase in human tissues and extracellular fluids. Acta Physiol Scand Suppl 492: 19-23.

13. Paglia DE, Valentine WN (1967) Studies on the quantitative and qualitative characterization of erythrocyte glutathione peroxidase. J Lab Clin Med 70(1): 158-169.
14. Niehans WG, Samuelson BAS (1968) Rapid method for the estimation of malondialdehyde, European. Journal of Biochemistry 6: 126-128.

15. Aho K, Harmsen P, Hatano S, Marquardsen J, Smirnov VE, et al (1998) Cerebrovascular disease in the community: results of a WHO collaborative study. Bull World Health Organ 58(1): 113-130.

16. Cook NL, Vink R, Helps SC, Manavis J, van den Heuvel C (2010) Transient Receptor Potential Melastatin 2 Expression is Increased Following Experimental Traumatic Brain Injury in Rats. Journal of Molecular Neuroscience 42(2): 192-199.

17. Noori S (2012) An Overview of Oxidative Stress and Antioxidant Defensive System. Open Access Scientific Reports 1(8): 1-9.

18. Rabec GV, Pierce RC (1994) A vitamin as neuromodulator. Ascorbate release into the extracellular fluid of the brain regulates dopaminergic and glutamertagic transmission. Prog Neurobiol 43(6): 537-565.

19.Eghwrudjakpor PO, Allison AB (2010) Oxidative stress following traumatic brain injury: enhancement of endogenous antioxidant defense systems and the promise of improved outcome. Niger I Med 19(1): 1421.

20.Inci S, Ozcan OE, Kilinic K (1998) Time-level relationship for lipid peroxidation and the protective effect of $\alpha$-tocopherol in experimental mild and severe brain injury. Neurosurgery 43(2): 330-335.

21. Ramirez-Tortosa C, Andersen ØM, Cabrita L, Gardner PT, Morrice PC, et al (2001) Anthocyanin-rich extract decreases indices of lipid peroxidation and DNA damage in vitamin E-depleted rats. Free radical Biology and Medicine 31(9): 1033-1037.

22.Lu C, Mattson MP (2001) Dimethyl sulfoxide suppresses NMDA- and AMPA-induced ion currents and calcium influx and protects against excitotoxic death in hippocampal neurons. Exp Neurol 170(1): 180-185.

23. Karaca M, Kilici E, Yazici B, Demir S Torre JC (2002) Ischemic stroke in elderly patients treated with free radical scavenger-glycolytic intermediate solution: a preliminary pilot trial. Neurol Res 24(1): 73-80. 